exact volume of diluent and a closed system transfer device (CSTD). Nurses just have to dilute the solution into the bag under a laminar air-flow hood using the CSTD. Although 2-part syringe methods were found in the literature, 3-part syringes with limited contact between the elastomeric tip and busulfan solution (reference 62.8426, Codan) were chosen because leaks were observed with the 2-part syringes during the technical study. PhaSeal devices: Injector to close the syringes and a Connector-Luer for infusion bags were selected as CSTDs. All these devices are polycarbonate free.

7 new kits were prepared for a period of 8 days without contact. The results of the evaluation show that nurses and physicians ( $n=14$ ) were overall dissatisfied by the previous protocol (neither good nor bad: $35.7 \%$, bad: $21.4 \%$ and very bad: $35.7 \%$ ) while the majority preferred the new one (very satisfied: $28.6 \%$, satisfied: $42.9 \%$, neither good nor bad $7.14 \%$, no response: $21.4 \%$ ). Overall nurses and physicians answered that new modalities limit the risk of dose errors (93\%) and occupational exposure (86\%).

Conclusions Implementing this procedure has improved handling practise with good satisfaction.

No conflict of interest.

\section{TCH-029 OUTCOMES EVALUATION OF AN INTERNATIONAL WORKGROUP ON ROBOTICS: A MULTICENTRE STUDY}

doi:10.1136/ejhpharm-2013-000276.220

${ }^{1} \mathrm{C}$ Bufarini, 'A Marinozzi, ${ }^{2} \mathrm{C}$ Masini, ${ }^{3} \mathrm{M}$ Milani, ${ }^{3} \mathrm{E}$ Omodeo Salè, ${ }^{4} \mathrm{R}$ La Russa, ${ }^{4} \mathrm{~B}$ Bultrini, ${ }^{4} \mathrm{~L}$ Ragni, ${ }^{5} \mathrm{~A}$ Yaniv, ${ }^{5} \mathrm{~S}$ Calabrese. 'AO Ospedali Riuniti, Pharmacy, Ancona, Italy; ${ }^{2}$ Romagna Cancer Institute, Pharmacy, Meldola (FC), Italy; ${ }^{3}$ European Institute of Oncology, Pharmacy, Milan, Italy; ${ }^{4}$ S. Camillo Hospital, Pharmacy, Rome, Italy; ${ }^{5}$ Cleveland Clinic, Pharmacy, Cleveland (OH), USA

Background The growing demand for patient and operator safety in anticancer drug compounding led to an increasing demand for automation. As 18 hospitals have now introduced APOTEC Achemo, it became necessary to set up a round table at which all users could share their knowledge and expertise. Therefore, in 2009 a workgroup on robotics (named APOTECA Community) was established. Every year its members meet to share their needs with the manufacturer and assemble new ideas. The annual system upgrade is a consequence of the meeting. 160 new requirements, merged into 4 upgrades, have been collected up to now. The requirements can be classified into the following main topics: a friendlier user interface; software integration with medical health records; higher productivity.

Purpose To assess the results in terms of productivity following the 2012 upgrade within the APOTECA Community

Materials and Methods Five oncology pharmacies were selected for this study: University Hospital of Ancona, European Institute of Oncology, Romagna Cancer Institute, S. Camillo Hospital of Rome, Cleveland Clinic. The abovementioned pharmacies were monitored before and after the upgrade, as far as the monthly productivity with APOTEC Achemo is concerned.

Results The 5 hospitals together prepared an average of 4150 preps/month before the 2012 upgrade, while 6000 preps/month was surpassed after the installation. Productivity showed an average increase of $46 \%$, ranging from $11 \%$ to $67 \%$. This variability is closely correlated with the best practise and has been analysed for each case.

Conclusions The creation of a round table where the APOTECAchemo users share experiences and discuss best practise is playing an essential role in the continuous improvement of this innovative technology. The progress recorded after the latest upgrade in terms of productivity $(+46 \%)$ is only one example of this powerful tool.

No conflict of interest.

\section{TCH-030 PET/CT IMAGING WITH [11C]CHOLINE AS A RADIOPHARMACEUTICAL FOR THE DETECTION OF RECURRENT PROSTATE CANCER: A RELIABLE PRODUCTION METHOD AND QUALITY CONTROL}

doi:10.1136/ejhpharm-2013-000276.221

${ }^{1} \mathrm{M}$ Riondato, ${ }^{1} \mathrm{~A}$ Democrito, ${ }^{2} \mathrm{MC}$ Bagnara, ${ }^{3} \mathrm{M}$ Massollo, ${ }^{3} \mathrm{GM}$ Sambuceti. ${ }^{1} / R C C S$ San Martino - IST, Nuclear Medicine Radiopharmacy, Genoa, Italy; ${ }^{2}$ RCCS San Martino IST, Health Physics, Genoa, Italy; ${ }^{3}$ RRCCS San Martino - IST, Nuclear Medicine, Genoa, Italy

Background PET/CT Imaging with the radiopharmaceutical [11C]-choline has become a useful tool in the detection of prostate cancer, mainly used in the assessment of treated patients presenting rising PSA and negative response after conventional imaging procedures. Tracer uptake on tumoral tissues is correlated to an increased synthesis of membrane substrates: [11C]-choline is trapped by phosphorylation taking part on phosphatidylcholine turnover. The sensitivity of this diagnostic method (almost 100\%) is greater than CT or PET-[18F]FDG implying the superiority of the PET-choline procedure. PET-choline was first investigated in the late 1990s although no specific monographs are included in main Pharmacopoeias. The use of this powerful tracer is now based on Clinical Trials but, on September 2012, the FDA approved the production and use of 'Choline C11 Injection' to help the detection of recurrent prostate cancer.

Purpose To define the key role the pharmacist plays in the preparation of [11C]-choline IMPD for Clinical Trials, presenting the tracer production in the details. Quality Control for characterising the final product and releasing it as 'solutio iniectabilis' are also described.

\section{Materials and Methods}

cyclotron (Eclipse, Siemens)

Automatized synthesiser (ModularLab, Eckert Ziegler)

GMP grade reagents and disposables

[11C]labelling based on 'wet' methylation chemistry

Results [11C]carbon dioxide (50 GBq) was produced by cyclotron and delivered to the synthesiser placed in our radiopharmacy. Carbon dioxide was first reduced to methyl iodide, then dimethyl-aminoethanol was [11C]-methylated. Finally the product was purified and filtered obtaining $15 \mathrm{GBq}$ of sterilised [11C]-choline (16 min total time and $30 \%$ yield). Radiochemical purity was higher than $98 \%$ and other CQs were performed in accordance with EPh [18F] FDG monograph.

Conclusions Due to the short half-life decay (20 min) [11C]choline production must be performed in PET facilities with on-site cyclotron and radiopharmacy. We presented a reliable and safe method for producing [11C]-choline for 3-4 patients' PET scans.

No conflict of interest.

\section{TCH-031 PHARMACIST EXPERIENCE IN CONTINUING IMPROVEMENT OF THE AUTOMATIC SYSTEM}

doi:10.1136/ejhpharm-2013-000276.222

'M Milani, 'V Sirna, ${ }^{2} \mathrm{~V}$ Rosini, ${ }^{2} \mathrm{D}$ Paolucci, ${ }^{1} \mathrm{E}$ Omodeo Salè. ${ }^{1}$ European Institute of Oncology, Pharmacy, Milan, Italy; ${ }^{2}$ Loccioni, Humancare, Moie di Maiolati (AN), Italy

Background An annual meeting between the manufacturer of APOTECAchemo and all the users is held to share experience and discuss suggestions for best practise. The feedback collected during the meeting forms the basis for the next system upgrade aimed at increasing performance. Some improvements that we suggested were included in the 2012 upgrade and improved our productivity: a new procedure for reconstitutions; an extemporaneous picking list; faster communication between the management software and the robot; a more efficient vision system for identifying labels. 